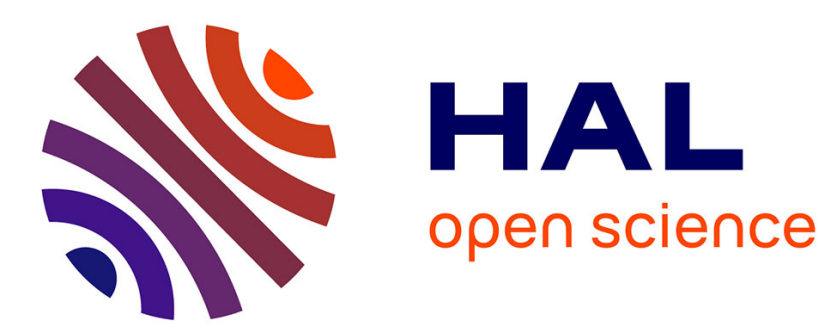

\title{
Spin effects in quasi-one-dimensional conductors under magnetic field
}

\author{
G. Montambaux, M. Héritier, P. Lederer
}

\section{To cite this version:}

G. Montambaux, M. Héritier, P. Lederer. Spin effects in quasi-one-dimensional conductors under magnetic field. Journal de Physique Lettres, 1984, 45 (11), pp.533-542. 10.1051/jphyslet:019840045011053300 . jpa-00232378

\section{HAL Id: jpa-00232378 https://hal.science/jpa-00232378}

Submitted on 1 Jan 1984

HAL is a multi-disciplinary open access archive for the deposit and dissemination of scientific research documents, whether they are published or not. The documents may come from teaching and research institutions in France or abroad, or from public or private research centers.
L'archive ouverte pluridisciplinaire HAL, est destinée au dépôt et à la diffusion de documents scientifiques de niveau recherche, publiés ou non, émanant des établissements d'enseignement et de recherche français ou étrangers, des laboratoires publics ou privés. 
Classification

Physics Abstracts

$72.15 \mathrm{~N}-74.10-75.25$

\title{
Spin effects in quasi-one-dimensional conductors under magnetic field
}

\author{
G. Montambaux, M. Héritier and P. Lederer \\ Laboratoire de Physique des Solides $\left({ }^{+}\right)$, Université Paris-Sud, Centre d'Orsay, \\ 91405 Orsay, France
}

(Reçu le 13 février 1984, accepté le 5 avril 1984)

\begin{abstract}
Résumé. - Nous étudions l'effet du champ magnétique sur la stabilité relative des phases onde de densité de spin, onde de densité de charge, métallique ou supraconductrice dans les métaux quasiunidimensionnels. Nous commençons par traiter le cas du gaz d'électrons unidimensionnel (modèle de $g$-ologie) par une méthode de groupe de renormalisation multiplicative à deux fréquences de coupure. Les couplages tridimensionnels sont ensuite schématisés par le découplage des canaux " Cooper " et " zero son " qui interfèrent à une dimension. Pour certaines valeurs des interactions et en négligeant les effets diamagnétiques, nous avons trouvé qu'une phase " onde de densité de spin » est stabilisée par le champ. Nous discutons le cas des composés (TMTSF) ${ }_{2} X$.

Abstract. - We study the effect of the magnetic field on the relative stability of charge density wave, spin density wave, metallic or superconducting states of quasi-one-dimensional superconductors. First, we study the case of the one-dimensional electron gas in the $g$-ology model with a multiplicative renormalization group method with two cut-off energies. Then, three-dimensional couplings are simply taken into account by the decoupling of the Cooper and zero sound channels which interfere in one dimension. For a given set of interactions and neglecting diamagnetic effects, we find that a SDW state is stabilized under field. Applicability to the (TMTSF) ${ }_{2} \mathrm{X}$ compounds is discussed.
\end{abstract}

\section{Introduction.}

The compounds of the family (TMTSF) ${ }_{2} \mathrm{X}$ exhibit a competition between superconductivity and magnetism, revealed by many experiments [1]. As the temperature is decreased in $(\mathrm{TMTSF})_{2} \mathrm{PF}_{6}$, a transition from a metallic state to an insulating magnetic state occurs at ambient pressure, but a superconducting state appears at high enough pressure. The magnetic and superconducting phases are so close together that a moderate pressure of about $8 \mathrm{kbars}$ is enough to stabilize a superconducting phase against the magnetic phase observed at ambient pressure. (TMTSF) ${ }_{2} \mathrm{ClO}_{4}$ is already superconducting at ambient pressure, but a quenching of anions disorder is able to suppress the superconductivity and to stabilize a magnetic state. Such a vicinity of magnetic and superconducting phases in these strongly anisotropic compounds

$\left(^{+}\right)$Laboratoire associé au C.N.R.S. 
is reminiscent of the properties of the one-dimensional electron gas for which the most thoroughly studied model is the $g$-ology model [2]. In one dimension, the electron gas is unstable, at zero temperature, against the formation of a spin density wave (SDW), a charge density wave (CDW) or singlet or triplet superconductivity (SS or TS), depending on the relative strengths of the Fourier components of the electron-electron interaction, particularly around wave vectors $q=0, q=2 k_{\mathrm{F}}$ and $q=4 k_{\mathrm{F}}\left(k_{\mathrm{F}}\right.$ is the Fermi wave vector of the electron gas). However, a transverse coupling between the one-dimensional chains is necessary to stabilize a long range order.

Depending on the strength of this transverse coupling, two different pictures have been proposed to describe the (TMTSF) ${ }_{2} \mathrm{X}$ family. In the first, the transverse coupling is much smaller than the longitudinal one; these organic metals should be considered as quasi-one-dimensional conductors in which large quantum or thermal fluctuations strongly influence all the physical properties up to temperatures much higher than the three-dimensional ordering temperature [1]. In the second, the coupling is so large that the metals should be considered as anisotropic, but essentially three-dimensional conductors in which the fluctuations are unimportant except in the close vicinity of the transition temperature [3]. A number of arguments has been put forward in favour of each of these pictures. At the present time, none of them seems to suffer from internal inconsistency. However, one must be able to discriminate between the two pictures by resorting to finer details of the theories, or by considering more complete experimental data. Magnetic field effects would seem to be good candidate for such a discussion. A magnetic field has actually been applied in the superconducting phase of (TMTSF) ${ }_{2} \mathrm{ClO}_{4}$ (at ambient pressure) and in the metallic phase of (TMTSF) ${ }_{2} \mathrm{PF}_{6}$ (at about 7-9 kbars) [1]. These metals are known to be type II superconductors with strongly anisotropic upper critical fields ranging from less than $1 \mathrm{kG}$ in the $c^{*}$ direction to more than $50 \mathrm{kG}$ in the $a^{*}$ direction. This diamagnetic suppression of the superconductivity by the magnetic field will not be discussed in detail here. In this work, we are mainly interested in the sharp transition from normal metal to a semi-metallic SDW state which is observed when the field is applied in the $c^{*}$ direction at an onset field of the order of $50 \mathrm{kG}$ [4-7].

In (TMTSF) ${ }_{2} \mathrm{ClO}_{4}$ as in (TMTSF) ${ }_{2} \mathrm{PF}_{6}$, further increase of the field enlarges the temperature range of existence of this phase. In the anisotropic three-dimensional metal picture, no clear explanation has been proposed at present time, as far as we know, for such a transition under a field much smaller than the Fermi energy. We have not explored this way further. In the quasione-dimensional metal picture, the theory of the effects of an applied magnetic field on the phase diagram remains to be done. Given the interest raised up by these magnetic field experiments, the absence of clear explanation and the state of the controversy about the nature of the organic conductors, such a study seems an obvious necessity. Surprisingly enough, very few papers until now have been devoted to the magnetic field effects on a 1-D electron gas. In fact, one paper deals with a restricted version of the $g$-ology model, which considers no coupling constant anisotropy and no Umklapp term [8]. A comment of this paper is found in the review paper by Solyom [2]. Another one studies the strong coupling region using the exact Luther-Emery solution $[9,10]$.

The purpose of this letter is two-fold : first, we indicate, on the basis of a renormalization group analysis, how the singularities in the response functions of the one-dimensional electron gas in the $g$-ology model, are modified by an applied magnetic field; then, we consider the effects of those modifications on the phase transitions of quasi-one-dimensional conductors when a transverse electron transfer between the one-dimensional chains decouples the CDW, SDW and Cooper pair channels at low temperature. Secondly, we discuss whether such a theory could explain the field-induced transition to a SDW semi-metallic state in the (TMTSF) ${ }_{2} \mathrm{X}$ metals.

A more detailed account of this work is in preparation [11]. 


\section{Magnetic field effects on the one-dimensional electron gas.}

The electron gas is described by a simple Luttinger model in which the one-electron dispersion relation is linearized around the Fermi wave vector $k_{\mathrm{F}}$. A bandwidth cut-off $E_{0}$ is introduced. The electrons interact through backward scattering, with coupling strength $g_{1}$ (the momentum transfer $q \simeq 2 k_{\mathrm{F}}$ ) and forward scattering, with coupling strength $g_{2}(q \simeq 0)$. In reference [8], the response functions were calculated in the parquet approximation. Here, we start with the same Luttinger model in the $g$-ology picture, which is generalized by introducing different coupling strengths for electrons with parallel spins, $g_{1 \|}, g_{2 \|}$ or antiparallel spins $g_{1 \perp}, g_{2 \perp}$. We also consider an Umklapp scattering $g_{3}\left(q \simeq 4 k_{\mathrm{F}}\right)$ which may be important in the case of a half-filled band (a case of particular interest since it corresponds to a whole class of materials [12]). The general technique used is the multiplicative renormalization group method elaborated by Menyhard and Solyom [13] for the same problem in the absence of a magnetic field. However, this method must be conveniently modified to take care of a second energy scale, besides the bandwidth cut-off $E_{0}$, introduced by the field, namely the Zeeman energy splitting $\Delta$ between up-spins and down-spins [2]. The one-electron dispersion relation in a magnetic field becomes :

$$
\varepsilon_{\sigma}(k)=\varepsilon(k)+\sigma \frac{\Delta}{2}=v_{\mathrm{F}}\left(|k|-k_{\mathrm{F}_{\sigma}}\right) \quad \sigma=+1 .
$$

This is the only effect of the field in one dimension. In particular, diamagnetism disappears, as can be shown by choosing the vector potential perpendicular to the chain. In zero field, all the vertex diagrams in $g_{1}$ and $g_{2}$ diverge as $\log \omega / E_{0}$ as the frequency $\omega \rightarrow 0$, because of the one-dimensional Fermi surface. In the presence of the field, the vertex diagrams in $g_{1 \|}, g_{2 \|}$ and $g_{2 \perp}$ still diverge as $\log \omega / E_{0}$, but this is not true anymore for the $g_{1 \perp}$ diagram because of the different values of the Fermi wave vector $k_{\mathrm{F} \uparrow}$ and $k_{\mathrm{F} \downarrow}$ for up- and down-spins : if the two incoming electrons with antiparallel spins are chosen on the Fermi surface, both of them cannot remain on the Fermi surface after a backward scattering without violating the momentum conservation. For that reason, this diagram behaves like $\log \Delta / E_{0}$, when $\omega<\Delta$.

First, we write a perturbation expansion, up to second order in the $g$ 's, for the vertices corresponding to the scattering of parallel spin electrons $\Gamma_{\|}$, the backward scattering of antiparallel spin electrons $\Gamma_{1 \perp}$, the forward scattering of antiparallel spin electrons $\Gamma_{2 \perp}$ and the Umklapp scattering $\Gamma_{3}$. As discussed above, these expansions involve terms proportional to $\log \omega / E_{0}$ and others proportional to $\log \Delta / E_{0}$. Since we are interested in physically available values of the field, $\Delta / E_{0} \ll 1$ and in the behaviour of the system at vanishing frequency $\omega \rightarrow 0$, the perturbation expansions are not valid. We are left with a problem with two logarithmic singularities, one in $\log \omega$, which is well known, the other in $\log \Delta$. We must set up a double multiplicative renormalization group procedure [11]. The two natural cut-offs to consider are $E_{0}$ and $\Delta$. Then, the only choice of two scaling parameters which can be varied independently is $\Delta / E_{0}$ and $\omega / \Delta$. The perturbation expansions for the vertices can be written as a function of these two scaling parameters. Let us write, for example, two of them [11], in $\pi v_{\mathrm{F}}$ units,

$$
\begin{aligned}
& \Gamma_{\|}=g_{1 \|}-g_{2 \|}+\frac{g_{1 \perp}^{2}+g_{3}^{2}}{2} \log \frac{\Delta}{E_{0}}+\frac{g_{3}^{2}}{2} \log \frac{\omega}{\Delta} \\
& \Gamma_{1 \perp}=g_{1 \perp}+g_{1 \perp}\left(g_{1 \|}-g_{2 \|}+g_{2 \perp}\right) \log \frac{\Delta}{E_{0}}+\frac{1}{2} g_{1 \perp}\left(g_{1 \|}-g_{2 \|}+g_{2 \perp}\right) \log \frac{\omega}{\Delta} .
\end{aligned}
$$

Let us recall that the vertex $\Gamma_{\|}$is calculated when all external momenta lie on the Fermi surface so that the total incoming momentum is 0 . This is not the case for $\Gamma_{1 \perp}$ where this total momentum is $\pm\left(k_{\mathrm{F} \uparrow}-k_{\mathrm{F} \downarrow}\right)$. This choice of external variables is dictated by the response functions. These 
diverge at a given wave vector (Table I). The perturbation expansion for a response function is then performed for this wave vector. Thus, it involves the calculation of vertices with a well defined set of external variables.

Table I. - Critical indices for response function power-law singularities, in the case where $g_{1 \perp}^{*}=0$.

\begin{tabular}{|c|c|c|}
\hline Type of instability & Wave vector of instability & Critical index \\
\hline CDW & $2 k_{\mathrm{F} \uparrow}$ or $2 k_{\mathrm{F} \downarrow}$ & $\frac{g_{1 \|}^{*}-g_{2 \|}^{*}+g_{3}^{*}}{2}$ \\
$1-\mathrm{SDW}$ & $2 k_{\mathrm{F} \uparrow}$ or $2 k_{\mathrm{F} \downarrow}$ & $\frac{g_{1 \|}^{*}-g_{2 \|}^{*}-g_{3}^{*}}{2}$ \\
$\mathrm{t}-\mathrm{SDW}$ & $k_{\mathrm{F} \uparrow}+k_{\mathrm{F} \downarrow}=2 k_{\mathrm{F}}$ & $-\frac{g_{2 \perp}^{*}}{2}+\frac{g_{3}^{*}}{2}$ \\
$\mathrm{SS}$ & $\pm\left(k_{\mathrm{F} \uparrow}-k_{\mathrm{F} \downarrow}\right)$ & $\frac{g_{2 \downarrow}^{*}}{2}$ \\
$\left(S_{z}=0\right)-\mathrm{TS}$ & $\pm\left(k_{\mathrm{F} \uparrow}-k_{\mathrm{F} \uparrow}\right)$ & $\frac{g_{2 \downarrow}^{*}}{2}$ \\
$\left(S_{z}=1\right)-\mathrm{TS}$ & 0 & $-\frac{g_{1 \|}^{*}-g_{2 \|}^{*}}{2}$ \\
\hline
\end{tabular}

Obviously, one should find again the zero field expressions by putting $\omega=\Delta$, as observed. Since we have a logarithmic problem in $\Delta / E_{0}$, we can derive a Lie equation and make a multiplicative renormalization, keeping $\omega / \Delta$ constant and varying the scaling parameter $\Delta / E_{0}$ from 1 to its physical value. We call this renormalization, the field-renormalization because it relates the bare couplings (which would be measured in infinite field) to a finite field problem. From the remark above, one can see that the field-renormalization is the same as the frequency-renormalization in zero field, except that, in finite field, one stops the renormalization at finite value of the scaling parameter.

One obtains, in this way, field-renormalized couplings which depend logarithmically on $\omega / A$. These effective couplings are the starting point of the frequency-renormalization, in which the scaling parameter $\omega / \Delta$ is varied from 1 to $0, \Delta / E_{0}$ being kept constant. Such a two cut-off scaling has been used by Grest et al. [14]. In their case, the two energy scales are related to electron and phonon variables; our problem is simpler since our second cut-off does not introduce any new variable nor the subsequent difficulties described in reference [14].

In zero field, one obtains two sets of decoupled flows, corresponding to the separation of charge degrees of freedom, in the $\left(g_{1 \|}-2 g_{2}, g_{3}\right)$ plane, and spin degrees of freedom, in the $\left(g_{1 \|}, g_{1 \perp}\right)$ plane. In finite fields, the flows in the plane $\left(g_{1 \|}-2 g_{2}, g_{3}\right)$ are not altered. Indeed, the field only acts on the spin degrees of freedom, that is to say on the $\left(g_{1 \|}, g_{1 \perp}\right)$ plane. This is shown on figure 1. In the region $g_{1 \|} \geqslant\left|g_{1 \perp}\right|$, the fixed points line is always Tomonaga-like [2]: $g_{1 \perp}^{*}=0$, but $g_{1 \|}^{*}$, the value of $g_{1 \|}$ at the fixed point, is larger than that obtained in zero field. In particular, in the case of spin independent coupling $g_{1 \|}=g_{1 \perp}$, instead of $g_{1 \|}^{*}=0$ as in zero field, one obtains :

$$
g_{1 \|}^{*}(H)=\frac{g_{1}}{1-g_{1} \log \frac{\Delta}{E_{0}}} \geqslant 0 .
$$




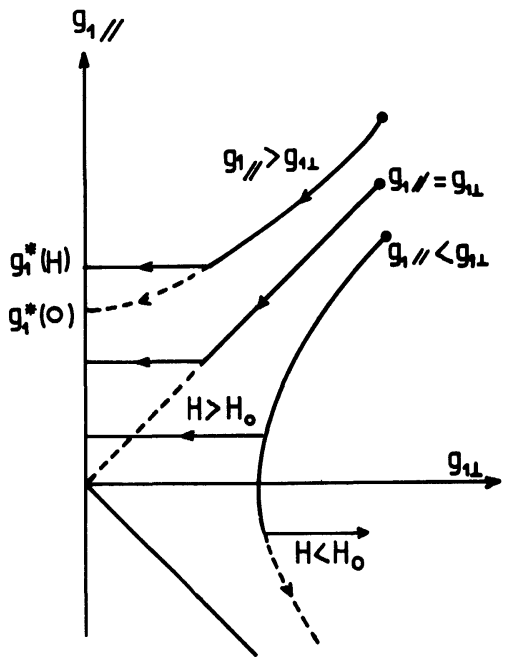

Fig. 1. - Typical scaling trajectories in the $\left(g_{1 \|}, g_{1 \perp}\right)$ plane under magnetic field (full line), when the starting points (bare couplings) are close to the line $g_{1 \|}=g_{1 \perp}>0$. Even in the particular case of spin independent couplings, the field breaks the $g_{1 \|}=g_{1 \perp}$ symmetry and the fixed point $(0,0)$ is removed to a finite value $\left(g_{1 \|}^{*}(H), 0\right)$. An interesting case occurs close to the line $g_{1 \|}=g_{1 \perp}$ when $g_{1 \|}<\left|g_{1 \perp}\right|$ : a field larger than a critical field $H_{0}$ can remove the fixed point from strong coupling values $\left(g_{1}<0\right)$ to the Tomonaga line.

The field induces different values for the renormalized couplings $g_{1 \|}^{*}$ and $g_{1}^{*}$, even though the bare couplings are not spin dependent. This behaviour can be understood qualitatively by observing that the renormalization of $g_{1 \|}$ is due to a $g_{1 \perp}^{2}$ term (two $g_{1 \perp}$ interactions are necessary). Therefore the locking of the $g_{1 \perp}$ processes at frequency smaller than $\Delta$ stops the renormalization of $g_{1 \|}$.

In the case $g_{1 \|}<\left|g_{1 \perp}\right|$, the important result is that a large enough field, larger than $H_{0}$, moves the fixed point from the strong coupling region to the Tomonaga region. If the starting point is close enough to the line $g_{1 \|}=g_{1 \perp}$, a simple, Kosterlitz-like [15] expression can be found for $H_{0}$ :

$$
H_{0} \simeq E_{0} \exp -\frac{A}{\left(g_{1 \perp}-g_{1 \|}\right)^{1 / 2}} \text { with } A=\frac{\pi}{2 \sqrt{2 g_{1}}} .
$$

Note that this field is related to the gap $\Delta_{\mathrm{KT}}$ in this region by $H_{0}=\sqrt{E_{0} \Delta_{\mathrm{KT}}}$ and that this result is different from the one obtained in the strong coupling limit [9].

From the above results, one can already deduce important properties of the phase diagram. Since the field does not have any effect on the $\left(g_{1 \|}-2 g_{2}, g_{3}\right)$ plane, it does not allow to cross the line $g_{1 \|}-2 g_{2}=g_{3}$, which separates the superconducting responses from the density wave responses, as far as the dominant singularities are concerned. However, in a field larger than $H_{0}$, one can pass from a CDW region to a SDW region, or from a SS one to a TS one.

For a complete discussion of the dominant and secondary singularities, we need to derive the response functions. First let us note that, when the spin anisotropy of the coupling constants is taken into account, one obtains different expressions for the SDW or triplet pairing response functions transverse to the field ( $t$ ) or longitudinal to the field (l). We have performed a double renormalization of the response functions in the same spirit as in the case of the vertices. We find power-law singularities as $\omega \rightarrow 0$, with critical indices given in table I. Here, the $g^{* ' s}$ are the 
fixed point values of the renormalized coupling constants. In the simple case where $g_{3}=0$ and $g_{i \|}=g_{i \perp}$, the field dependence of the critical exponents is the same as in reference [8]. From equation (3) and from the invariance of $g_{1}-2 g_{2}$, we obtain the field dependence of the critical exponents and, then, the phase diagram shown in figure 2 . Considering that the perturbative expansions in powers of the $g_{i}$ 's do not lead to reliable enough results in the strong coupling regime, we have limited this study to regions of attraction of fixed points corresponding to the weak coupling regime ("Tomonaga-like» regions i.e. $g_{1} \geqslant 0$ in the case of spin-independent couplings). This case seems relevant to the (TMTSF) ${ }_{2} \mathrm{X}$ family [12]. While the domains of dominant singularities are not affected by the field, SDW and $S_{z}=1$ triplet superconductivity invade the phase diagram, on both sides of the $g_{1}=2 g_{2}$ lines, as secondary singularities. They are algebraically weaker than the dominant ones, in contrast to the zero-field case where the differences were only logarithmic. As a result, if a physical mechanism is able to suppress the superconductivity but not the spin density wave, the SDW domain will be enlarged by the magnetic field. For example, the superconductivity of the organic metals is known to be very sensitive to impurities, irradiation defects or anion disorder.

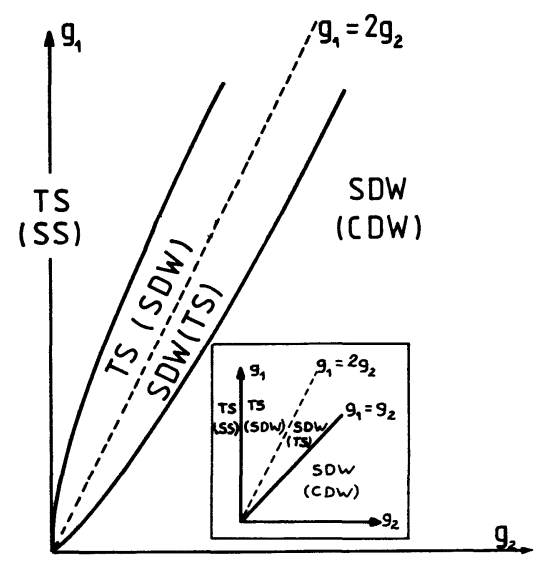

Fig. 2. - Phase diagram of the 1-D Fermi gas under magnetic field when $g_{3}=0$ and $g_{1}>0$. The response functions corresponding to the phases indicated in parentheses have a lower degree of divergence than others. The insert exhibits the case of an infinite field.

Just below the line $g_{1 \|}=\left|g_{1 \perp}\right|$, the renormalization treatment is known to be still appropriate to investigate the behaviour of the electron gas : it yields the Kosterlitz expression (4) i.e. critical field varying as $\sqrt{\Delta_{\mathrm{KT}}}$. Far from this line, this method becomes unvalid. The strong coupling region has been studied through the bosonization method [2] from which an exact solution is known for a particular value of the coupling constant $g_{1 \|}=-\frac{0}{5} \pi v_{\mathrm{F}}[10]$. The gap $\Delta$ is given by $\Delta_{\mathrm{LE}}=g_{1 \perp} / 2 \pi a, a$ being the lattice constant. Magnetic field effects have been studied in this region where a SDW instability can occur provided the field is larger than the gap $\Delta_{\mathrm{LE}}$ $[9,10]$.

\section{Quasi-one-dimensional case.}

In this section, we consider the case of one-dimensional chains, weakly coupled by electron transfer from chain to chain $t_{\perp}$. As soon as $t_{\perp}$ is finite, a diamagnetic orbital effect appears. Such an effect will not be discussed in detail here. We shall only consider two limiting cases : first, 
if the field is much smaller than the upper critical field $H_{\mathrm{c}_{2}}$, we consider that the superconducting phase is not affected by the orbital diamagnetism. Such a situation could be realized for not too small values of the field if it is parallel to the one-dimensional chain (direction of maximum $\boldsymbol{H}_{\mathrm{c}_{2}}$ ). In the second case, we consider fields high enough to destroy, not only the superconducting long range order $\left(H>H_{\mathrm{c}_{2}}\right)$, but also the short range order, which may demand much higher values. In such a case, we approximate the free energy by that of a completely disordered metal. For moderate values of the field, such an approximation is certainly more valid when the field is parallel to the direction of smallest transfer integral.

In three dimensions, the ordering temperatures are moved to finite values : as the temperature is decreased below a cross-over temperature $T_{\mathrm{co}}$, the one-dimensional behaviour becomes a three-dimensional one. The interference between the Cooper and the zero sound channels is destroyed by the finite value of $t_{\perp}$ and the various instability channels decouple, leading to finite values of the ordering temperature. This effect is described here by using the very simple and crude decoupling procedure of Prigodin and Firsov $[12,16]$ which, in fact, amounts to treating the instabilities by RPA equations with coupling constants $g_{i}$ renormalized by one-dimensional equations : the $g_{i}$ 's are the solutions of the one-dimensional scaling equations when $T=T_{\text {co }} \simeq$ $t_{\perp} / \pi$ (if many-body effects on the electron transfer from chain to chain are ignored). This approximation neglects the details of warped Fermi surface nesting. This procedure can be generalized in the case where a magnetic field is applied.

Consider first the case where $H<H_{\mathrm{c}_{2}}$, i.e., the case of a field parallel to the one-dimensional chain. From figure 2, one can see that the superconductivity-SDW line is not affected by the field in one dimension; therefore the ordering temperatures of both instabilities in quasi-1-D conductors vary by the same amount in a magnetic field and the phase diagram is not modified (as long as the orbital effects are neglected).

Consider now the case $H \gg H_{\mathrm{c}_{2}}$. We are interested here by the stability of the transverse SDW phase compared to the disordered metallic phase, which is increased by the field, while the stability of the longitudinal SDW phase is decreased. Two situations must be distinguished :

- when $H>T_{\text {co, }}$, the one-dimensional renormalization involves two steps, as explained in the previous section : $\Delta / E_{0}$ is varied from 1 to its physical value; then the scaling of $T / \Delta$ is performed down to the cross-over value $T_{\mathrm{co}} / \Delta$. In such a case the $g_{i}$ 's depend on the field;

- when $H<T_{\text {co }}$, the field effect is suppressed by the 1-D renormalization, $H / E_{0}$ being scaled down to $T_{\mathrm{co}} / E_{0}$. Since the RPA equations for transverse SDW response do not depend on $H$, the field is too weak to influence the renormalization of the coupling constants by the one-dimensional fluctuations. Still in the case $g_{3}=0$, we get the following result for the SDW ordering temperature

where

$$
T_{\mathrm{SDW}}(H)=T_{\mathrm{co}} \exp -\frac{2}{\tilde{g}_{2}(H)}
$$

$$
\tilde{g}_{2}(H)=g_{2}-\frac{g_{1}}{2}+\frac{\frac{g_{1}}{2}}{1-g_{1} \log \max \left(\frac{T_{\mathrm{co}}}{E_{0}}, \frac{\Delta}{E_{0}}\right)} .
$$

This procedure amounts to a three cut-off renormalization, the successive scaling parameters being

$$
\begin{array}{lll}
\frac{H}{E_{0}}, \frac{T_{\mathrm{co}}}{H}, \frac{T}{T_{\mathrm{co}}} & \text { if } & H>T_{\mathrm{co}} \\
\frac{T_{\mathrm{co}}}{E_{\mathrm{o}}}, \frac{H}{T_{\mathrm{co}}}, \frac{T}{H} & \text { if } & T_{\mathrm{co}}>H>T .
\end{array}
$$


The effective coupling $\tilde{g}_{2}(H)$ increases with the field. Therefore, if a SDW is stable in zero field, the ordering temperature increases with the field. If this is not the case (i.e. $\left.\tilde{g}_{2}(H=0)<0\right)$, a SDW is stabilized at the threshold field $H_{\mathrm{T}}$ for which $\tilde{g}_{2}(H)$ changes its sign. The behaviour of the ordering temperature near the threshold field is given by an increasing function of $H$

$$
T_{\mathrm{SDw}}(H)=T_{\mathrm{co}} \exp \frac{B}{H_{\mathrm{T}}-H}
$$

where $B$ is a positive constant which we do not write explicitly. Let us emphasize that the threshold field cannot be smaller than $T_{\mathrm{co}}$. However, this lower limit can be reached only in the case where the SDW is almost stable in zero-field. In the $g$-ology picture, this means that the zero-field ground state is superconducting but very close to the $g_{1}-2 g_{2}=g_{3}$ line. If we assume, as in reference [12], a monotonic pressure variation of $g_{3}$ (with $g_{1}$ and $g_{2}$ independent of pressure) a phase diagram as that of figure 3 can be derived.

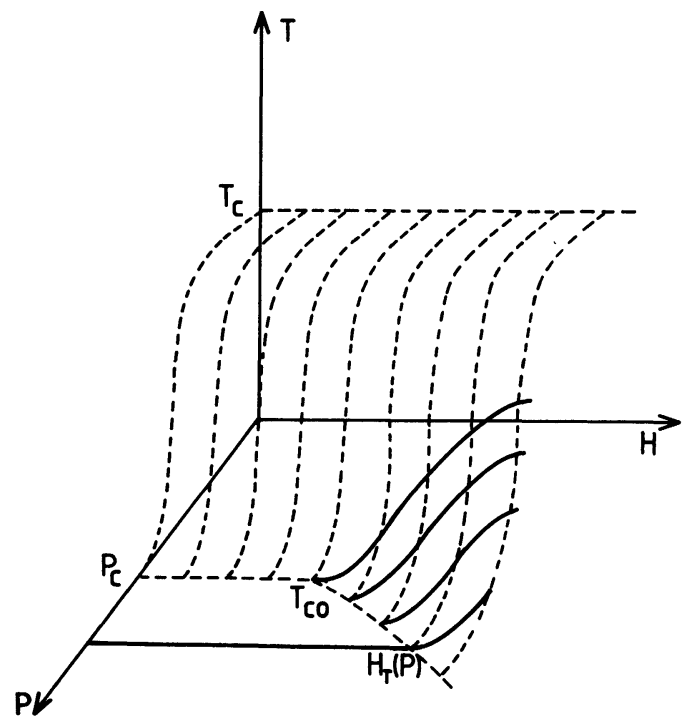

Fig. 3. - Typical phase diagram for the quasi-1-D Fermi gas in magnetic field. Assuming a decreasing variation in pressure for $g_{3}$, an essential singularity at low $T$ has been derived for the transition between metallic (N) and SDW phases (dashed line). Above $P_{\mathrm{c}}$, the field stabilizes the SDW phase (full line).

\section{Discussion of the experimental results.}

It is tempting to ascribe the field induced transition to a semi-metallic magnetic state observed in (TMTSF $)_{2} \mathrm{ClO}_{4}$ at ambient pressure and in $(\mathrm{TMTSF})_{2} \mathrm{PF}_{6}$ under a pressure of about $6.8 \mathrm{kbars}$ [4-7] to the effects discussed in this letter. According to the previous section, a magnetic field parallel to $c^{*}$, i.e. the direction corresponding to the smallest $H_{\mathrm{c}_{2}} \sim 1 \mathrm{kG}$ can stabilize a SDW if $H \gg H_{\mathrm{c}_{2}}$. Then, we expect that the SDW opens a gap at the Fermi level in the direction of smallest bandwidth, i.e. in the $c^{*}$ direction, but leaves small pockets of electrons and holes in the $(a, b)$ plane. Hence, the transition to a semi-metallic state, with a small number of charge carriers as measured by Hall effect [6], a sharp decrease of the electronic specific heat [4] and Shubnikov-de Haas oscillations [7] associated with closed orbits in the $(a, b)$ plane. 
The first fact that a correct theory must explain is the moderate value of the transition field (40-60 kG). Since in both compounds one may think that the superconducting and magnetic phases are almost degenerate, it is possible that the threshold field is close to its smallest possible value $T_{\text {co }}$, which depends on the transverse transfer integrals. Estimated values of $t_{b}$ range from $300 \mathrm{~K}$ [17] to $100 \mathrm{~K}$ [1]. $t_{c}$ is an order of magnitude smaller. If $t_{b}=300 \mathrm{~K}, T_{\text {co }} \simeq t_{b} / \pi$ which is larger by a factor of 20 than the observed threshold fields. If $t_{b}=100 \mathrm{~K}, t_{b} / \pi$ may be smaller than the mean-field ordering temperature. In such a case, Bourbonnais argued that the oneelectron value $t_{b} / \pi$ is reduced by many-body effects [18]. In the limit of low temperature

$$
T_{\text {co }} \sim \frac{t_{b}}{\pi}\left(\frac{t_{b}}{E_{0}}\right)^{\gamma}
$$

where the exponent $\gamma$ is of the order of 1 . Such an effect might account for the observed threshold fields but a confirmed value of $t_{b}=300 \mathrm{~K}$ would probably rule out such an explanation.

The observed anisotropy of the effect is an even more serious difficulty to deal with in this scheme. It seems that the relevant quantity in these experiments is the component of the field parallel to $c^{*}$ [19]. While this is expected in Shubnikov-de Haas experiments, since the closed orbits lie in the $(a, b)$ plane, this is more difficult to understand for $T_{\mathrm{SDw}}(H)$. We do expect a strong anisotropy in our picture for the following reason : the persistence of short range superconducting order decreases the stability of the SDW compared to the normal metallic state. Tunnelling experiments [20] have revealed the persistence of a pseudo gap with applied transverse fields up to $20 \mathrm{kG}$. It is possible that a field $\sim 50 \mathrm{kG}$ is able to destroy short range order only when parallel to $c^{*}$. (In the $b$-direction, the upper critical field $H_{\mathbf{c}_{2}}$ is larger by a factor of $\sim 15$.) However, we cannot understand, in our picture, that $T_{\text {SDw }}(H)$ does not seem no depend on the component of the field parallel to $b$. This fact needs experimental confirmation and, in any case, a more detailed theoretical treatment. Let us emphasize important weaknesses of our treatment in three dimensions : diamagnetic effects are taken into account only in as much as they suppress superconductivity. Interchain transverse hopping has been implicitly assumed to be isotropic; its main effect has been to decouple the divergent channels in one dimension. Obviously a quantitative theory of the observed spatial anisotropies needs a more refined approach. Likewise, the high field anomalies reported recently in reference [6] are completely out of the scope of our approach and require a detailed account of transverse hopping as well as diamagnetism. We conclude that we cannot state with certainty that our theory applies to (TMTSF) ${ }_{2} \mathrm{ClO}_{4}$ and $(\mathrm{TMTSF})_{2} \mathrm{PF}_{6}$. It would certainly apply to systems with a small enough $t_{b}$.

An interesting result of our theory is that a large enough magnetic field may change a CDW ground state into a SDW one. The conditions are a not too large bandwidth and a small spin anisotropy of the coupling constants. For example, for a bandwidth of $\sim 1 \mathrm{eV}$ and an anisotropy $\frac{g_{1 \perp}-g_{1 \|}}{g_{1}} \sim 5 \times 10^{-2}$ the critical field would be $H_{0} \sim 50 \mathrm{kG}$. It is possible that a compound of the $\mathrm{NbSe}_{3}$ family would be a good candidate in which one could observe this effect.

\section{Acknowledgments.}

We are grateful to S. Barišić, C. Bourbonnais, J. Friedel, P. Garoche, D. Jérome, M. Ribault and $\mathbf{H}$. J. Schulz for useful discussions. 


\section{References}

[1] Jérome, D. and Schulz, H. J., Adv. Phys. 31 (1982) 299 and references therein.

[2] SOlyom, J., Adv. Phys. 28 (1979) 201 and references therein.

[3] KWAK, J. F., J. Physique Colloq. 44 (1983) C3-839.

[4] Garoche, P., Brusetti, R., Jérome, D. and BechgaArd, K., J. Physique Lett. 43 (1982) L-147.

[5] TAKahashi, T., Jérome, D. and BechgaARd, K., J. Physique Lett. 43 (1982) L-565.

[6] Ribault, M., Jérome, D., Tuchendler, J., Weyl, C. and BechgaArd, K., J. Physique Lett. 44 (1983) L-953.

[7] Kwak, J. F., Schirber, J. E., Greene, R. L. and Engler, E. M., Mol. Cryst. Liq. Cryst. 79 (1982) 111.

[8] Gurgenishvili, G. E., Nersesyan, A. A. and Chobanyan, L. A., J.E.T.P. 46 (1977) 145.

[9] Japaridze, G. I. and Nersesyan, A. A., J. Low Temp. Phys. 37 (1979) 95.

[10] Luther, A. and Emery, V. J., Phys. Rev. Lett. 33 (1974) 589.

[11] Montambaux, G., Héritier, M. and Lederer, $P$., in preparation.

[12] Emery, V. J., Bruinsma, R. and Barišić, S., Phys. Rev. Lett. 48 (1982) 1039.

[13] Menyhard, N. and Solyom, J., J. Low Temp. Phys. 12 (1973) 529.

[14] Grest, G. S., Abrahams, E., Chui, S. T., Lee, P. A. and Zawadowski, A., Phys. Rev. B. 14 (1976) 1225.

[15] Kosterlitz, J. M., J. Phys. C 7 (1974) 1046.

[16] Prigodin, V. N. and Firsov, Yu. A., J.E.T.P. 49 (1979) 369.

[17] KWAK, J. F., Phys. Rev. B 26 (1982) 4789.

[18] Bourbonnais, C., preprint (1983).

[19] Azevedo, L. J., Schirber, J. E., Greene, R. L. and Engler, E. M., Physica B 108 (1981) 1183.

[20] Fournel, A., More, C., Roger, G., Sorbier, J. P., Delrieu, J. M., Jérome, D., Ribault, M. and BeChgaARD, K., J. Physique Lett. 42 (1981) L-445. 